\title{
Lesbianismo y literatura chicana: la construcción de una identidad/
}

Lesbianism and Chicana Literature:

Constructing an Identity

María Ángeles Toda Iglesia

Universidad de Sevilla

El artículo estudia la construcción de una identidad lésbica específica en la literatura de autoras chicanas, analizando sus raíces feministas, su empleo de diversas formas literarias, el papel de la teoría y la importancia del cuerpo, así como la presencia de los roles butch/femme, para después examinar los recursos empleados por las autoras para rechazar la persistente acusación de que su identidad constituye una traición a su comunidad. Dichos recursos incluyen el cuestionamiento de las políticas lesbófobas y antifeministas del movimiento chicano, el análisis de las relaciones familiares y la reescritura de mitos patriarcales mexicanos.

PALABRAS ClAVE: Literatura chicana; Lesbianismo.

The paper studies the construction of a specific lesbian identity in the literary production of Chicana lesbians, analyzing its feminist roots, its use of diverse literary forms, the role of theory and the importance of the body, as well as the presence of butch/femme roles; it later examines the devices employed by the authors to reject the persistent accusation that their identity constitutes a treason to their community. These devices include challenging the lesbophobic and antifeminist politics of the Chicano movement, analyzing family relations and rewriting Mexican patriarchal myths.

KEYwORDS: Chicana literature; Lesbianism. 
En 1971, la autora chicana Estela Portillo Trambley se convertía casi por accidente en la autora de la primera obra literaria chicana en la que aparecía una relación lésbica: The Day of the Swallows, publicada inicialmente en la revista minoritaria El Grito. Treinta años más tarde, el autor estadounidense Fred Reed, en una colección de artículos "políticamente incorrectos", inventa a una catedrática del "Departamento de Estudios de Lesbianas Chicanas y Transexuales Micronésicos", dando a entender la supuesta proliferación de las obras y los estudios de este tipo, a la vez que revela la viva hostilidad que continúan provocando. ${ }^{1}$ Pero a pesar de esta trivialización, ya en 1997 Hernández-Gutiérrez y Foster identificaban "el homoeroticismo" como uno de los seis temas centrales de la literatura chicana, ${ }^{2}$ y ya la obra de algunas autoras lesbianas chicanas, en particular Gloria Anzaldúa y Cherríe Moraga, ha pasado a incorporarse de modo ineludible no sólo al "canon" de la literatura chicana, sino a las líneas maestras del pensamiento lésbico y feminista, y en último término a los debates sobre identidad, sexualidad y etnicidad dentro de la sociedad globalizada. Como argumenta Catriona Rueda Esquibel, autora del primer estudio monográfico dedicado en exclusiva a la obra de las chicanas lesbianas, "Chicana lesbians are central to understanding Chicana/o communities, theories, and feminisms". ${ }^{3}$ En este trabajo quisiera reseñar de qué modo estas autoras, empleando una diversidad de recursos literarios, han construido, definido y representado una identidad lésbica chicana, y cómo la defienden, de modo recurrente, contra las acusaciones igualmente recurrentes de que dicha identidad supone una traición a sus raíces familiares, culturales y raciales.

Se hace necesario partir de dos definiciones iniciales, tanto del concepto "chicana" como del de "lesbiana". Por lo que se refiere al primero, las chicanas y los chicanos son personas de ascendencia mexicana residentes en los Estados Unidos de América; se trata de un término de sig-

1 Estela Portillo Trambley: The Day of the Swallows, en Roberto J. Garza (ed.): Contemporary Chicano Theatre. Fred Reed: Nekkid in Austin: Drop your Inner Child Down a Well, p. 2.

2 Manuel de Jesús Hernández-Gutiérrez y David William Foster (eds.): "Introduction," Literatura Chicana 1965-1995: An Anthology in English, Spanish and Caló, pp. ix-xvii, p. ix.

3 Catriona Rueda Esquibel: With her Machete in her Hand: Reading Chicana Lesbians, p. 3. Rueda Esquibel tiene también una valiosa bibliografía: Bibliography of 20th century Queer Chicana Fictions, accesible en http://online.sfsu.edu/ ktrion/Jotas (11-11-2009). Otros estudios recientes abarcan a otras lesbianas latinas, como el de Inmaculada Pertusa-Seva y Lourdes Torres: Tortilleras: Hispanic and U.S. Latina Lesbian Expression, Philadelphia. 
nificado reivindicativo, elegido frente a otras definiciones como méxicoamericanos o hispanos, por lo que es posible hablar, como lo hace Susan M. Guerra, de "proclamarse oficialmente chicana". ${ }^{4}$ El término abarca desde descendientes de los habitantes de estados como Texas, California, Utah o Nevada, que pertenecían originalmente a México y que pasaron a ser parte de los EEUU después de la guerra México-Americana - tras la derrota de México y el Tratado de Guadalupe-Hidalgo (1848), cuando casi un 50\% del territorio mexicano pasó a ser estadounidense- hasta inmigrantes de primera generación, legales o ilegales. En muchos casos, implica un origen multiracial (anglosajón, español, indígena, cuando no otros), con diversos grados de "visibilidad" física, y casi siempre un sentimiento de biculturalidad. ${ }^{5}$ Mucha parte de esta población se concentra en los estados del sudoeste (Arizona, Nuevo México, Texas, California...) pero también hay comunidades chicanas importantes en ciudades como Chicago o Detroit.

El término "lesbiana" parecería menos problemático, pero desde el cuestionamiento de los presupuestos patriarcales llevado a cabo por las activistas feministas y lesbianas a partir de los años 70, es una palabra que implica más que "2. Mujer homosexual", como lo define el Diccionario de María Moliner. ${ }^{6}$ Precisamente porque toda la escritura producida por autoras chicanas lesbianas parte de este contexto feminista, para la mayor parte de ellas el término es objeto de reflexión, y más allá de la simple orientación sexual implica una conciencia y un fuerte compromiso en cuanto a lo que significan estos vínculos sexuales y amorosos con otras mujeres. Ana Castillo, recogiendo este pensamiento radical lésbico-feminista, afirma que considera lésbica una relación que implica por parte de la mujer una decisión en cuanto a su forma de vida y un claro compromiso, esté o no manteniendo relaciones sexuales con otra mujer. ${ }^{7}$

4 Susan M. Guerra: "In the End (Al fin) we are all Chicanas (Somos todos chicanas): pivotal positions for change", en Gloria Anzaldúa y Ana Louise Keating (eds.): This bridge we call home: radical visions for transformation, pp. 181-190, p. 183.

5 Los ensayos autobiográficos "La Güera" (la rubia) de Cherríe Moraga y "La Prieta" (la negra), de Gloria Anzaldúa, ejemplifican esta diversidad en lo que se refiere al aspecto físico-racial de las chicanas. En Gloria Anzaldúa y Cherríe Moraga (eds.): This Bridge Called My Back: Writings by Radical Women of Color, pp. 24-33 y pp. 220-233.

6 María Moliner: Diccionario de uso del español. Edición abreviada..

7 Marta Navarro: "Entrevista con Ana Castillo", Carla Trujillo (ed.): Chicana Lesbians: The Girls Our Mothers Warned Us About, pp. 113-132, p. 119. 


\section{La construcción de una identidad}

En su antología de literatura lésbica, Lillian Faderman incluye a las chicanas, junto con otras mujeres de color, en la última de sus categorías ("post-lesbian-feminist literature"), marcada por el contexto de la teoría queer, los debates sobre ortodoxia sexual y el cuestionamiento de las limitaciones del feminismo "blanco", además de por la experimentación formal propia del postmodernismo. ${ }^{8}$ Como intentaré demostrar a continuación, esta clasificación me parece adecuada en cuanto a la inclusión de la variable de raza en el pensamiento feminista y respecto a las formas literarias empleadas, pero no en cuanto a los dos primeros aspectos, que tienen una presencia mínima. La obra de las lesbianas chicanas se enmarca más bien, a mi modo de ver, en un contexto clara y abiertamente feminista, con el objetivo claro de reivindicar, y desplazar a un lugar central, la experiencia de un grupo triplemente marginado, por su género, su raza y su orientación sexual, contribuyendo así a lo que Flora Alexander definió como el propósito fundamental del feminismo: llevar a cabo una reconceptualización a gran escala del mundo a fin de tener en cuenta la experiencia de las mujeres. ${ }^{9}$

En lo que se refiere a la experimentación formal, muchas autoras lesbianas chicanas emplean lo que Teresa de Lauretis denominó "fiction/theo$r y$ ", "a formally experimental, critical and lyrical, autobiographical and theoretically conscious, practice of writing-in-the-feminine that crosses genre boundaries". ${ }^{10}$ Esto sin duda no es ajeno al hecho de que un buen número de ellas son a la vez creadoras, críticas y/o profesoras, como ocurre en los casos de Gloria Anzaldúa (fallecida en 2004), Alicia Gaspar de Alba, Cherríe Moraga, Emma Pérez o Carla Trujillo, entre otras. Muchas autoras combinan e inventan géneros en sus obras individuales, mezclando autobiografía, poesía, teoría y narrativa, escribiendo diarios apócrifos o entrevistas imaginarias, o hilvanando novelas a partir de poemas en prosa; y además, varias de las obras esenciales para conocer la literatura lésbica chicana son hasta cierto punto creaciones comunitarias, compilaciones que abarcan todas estas modalidades de escritura. Muchas de ellas, debido a su

8 Lillian Faderman: Chloe plus Olivia: An Anthology of Lesbian Literature from the Seventeenth Century to the Present, pp. 689-693.

9 Flora Alexander: Contemporary Women Novelists, p. 7.

10 Teresa de Lauretis: "Sexual Indifference and Lesbian Representation", Henry Abelove et alt. (eds.): The Lesbian and Gay Studies Reader, pp. 141-158, p. 148. 
contexto bilingüe, alternan además inglés y español, incluso dentro de la misma obra, aunque la mayoría de la producción literaria se escribe en inglés, por motivos que merecerían un estudio más profundo de lo que podemos abarcar aquí. ${ }^{11}$

Esta experimentación es ineludible puesto que los patrones literarios tradicionales, ya ampliamente cuestionados por la crítica feminista como medios de expresar la subjetividad femenina, resultan aún menos aptos para hablar de una subjetividad y un deseo que, como señalaba Luce Irigaray, resulta simplemente incomprensible dentro de la lógica patriarcal. ${ }^{12}$ Tanto es así que para poder formular "lo que nunca pasó por sus labios", para citar el título de Cherríe Moraga, en los primeros escritos se ve una necesidad imperiosa de nombrar y nombrarse, de reivindicar, para transformarlos, los términos despreciativos, tanto en inglés como en español, con los que se ha denominado a las lesbianas. Sólo en la antología de Carla Trujillo, Chicana Lesbians, encontramos los poemas "Lesbiana", "Baby Dykes", "Trying to be Dyke and Chicana", que lo hacen desde el mismo título, y otros como "Intentarás imponerme", de Lidia Tirado White, que comienza con una enumeración de insultos ("Cachapera/ Manflora/ Jota/ Rara/ Maricona/ Anormal/ Tortillera/ Lesbiana/ Marimacha/ Andrógina/ Hombrecito/ Muchachito"), para concluir con una reivindicación de sí misma como "MUJER PARA SÍ". ${ }^{13}$ Una reivindicación que contrasta vivamente con el brutal acto de silenciamiento que lleva a cabo Josefa, una de las protagonistas de la ya citada The Day of the Swallows, que le corta la lengua a un niño que la ha visto mantener relaciones sexuales con su amante Alysea. ${ }^{14}$

Al haber comenzado a escribir dentro del contexto del feminismo lésbico, el cuestionamiento de los orígenes y la naturaleza de la identidad lesbiana, tan presente en alguno de los textos europeos clásicos de los siglos XIX y XX, no resulta en absoluto problemático; la legitimidad de la iden-

11 Gloria Anzaldúa, quizá de las escritoras más bilingües, da una idea de la complejidad implícita en el uso de las lenguas por los hablantes chicanos en "How to Tame a Wild Tongue", Borderlands/La frontera: The New Mestiza, pp. 75-86. Algunas de mis sugerencias sobre las connotaciones del uso del inglés y el español aparecen en María Ángeles Toda Iglesia: “¿Cuántas cervezas?: Dos versiones de un cuento de Alicia Gaspar de Alba” en Ramón Espejo et alt. (eds.): Critical Essays on Chicano Studies, pp. 245-254, p. 248.

12 Luce Irigaray, citada en Teresa de Lauretis, "Sexual Indifference", p. 142.

13 Cathy Arellano: "Lesbiana", pp. 12-13, Gina Montoya: "Baby Dykes", pp. 19-20, Natashia López: "Trying to be Dyke and Chicana", p. 84, Lydia Tirado White: "Intentarás imponerme", p. 84, en Trujillo (ed.), Chicana Lesbians.

14 Portillo Trambley, The Day of the Swallows, p. 241. 
tidad lésbica se da por hecho del modo que Faderman considera típico de la fase post-lesbianismo-feminista. Dicha identidad se vive como innata y percibida desde muy temprano, y varios textos reivindican de diversos modos la experiencia adolescente o incluso infantil de las protagonistas, dotándola de plena validez, muy en contra del mito de la supuesta "fase pasajera". Relatos como "Gulf Dreams" (1991) de Emma Pérez o "La Ofrenda" de Cherríe Moraga (1991) ${ }^{15}$ están narrados desde la perspectiva de adulta de la protagonista, que comprende y explica sus sentimientos y experiencias de adolescente: "I thought that writing this years later would finally release me from her. But I feel no reprieve. Not yet", afirma la narradora de "Gulf Dreams", declarando la persistencia de su amor adolescente. ${ }^{16}$ Moraga incide sobre esta continuidad entre el yo adulto y el yo adolescente en su drama Giving up the Ghost, donde aparecen en escena dos personajes distintos, la adolescente Corky y la adulta Marisa, que son la misma persona en dos etapas de su vida y se esclarecen mutuamente. Así, en su monólogo inicial, Corky relata sus intentos juveniles de actuar y sentirse como un chico, junto con su realización de la imposibilidad de hacerlo, y recibe el apoyo y la comprensión de Marisa, su yo adulto:

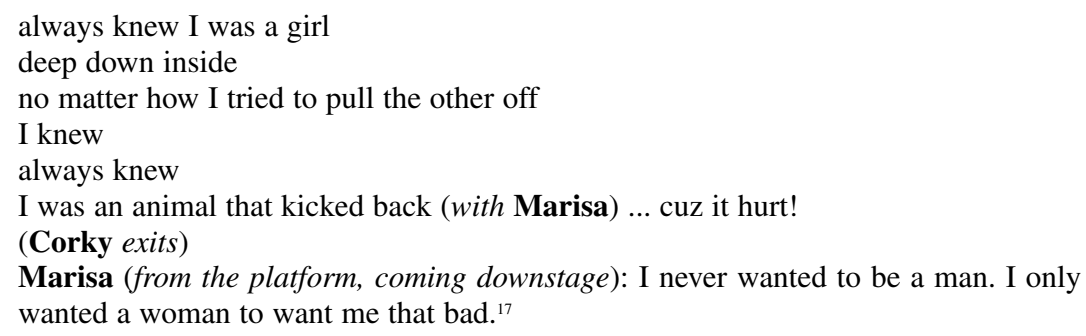

Marisa (from the platform, coming downstage): I never wanted to be a man. I only wanted a woman to want me that bad. ${ }^{17}$

Poemas, narrativas autobiográficas, e incluso los monólogos de la popular comediante Mónica Palacios insisten sobre el tema.

La importancia que se presta a la adolescencia remite a una de las tradiciones literarias en las que es posible enmarcar esta producción literaria: la del Bildungsroman. De modo más específico, Julián Olivares hace notar que el Bildungsroman es un género literario propio de los escritores y escritoras emergentes en EEUU, a menudo inmigrantes de primera o segunda

\footnotetext{
15 Emma Pérez: "Gulf Dreams”, en Trujillo (ed.), Chicana Lesbians, pp. 96-108 y Cherríe Moraga: "La Ofrenda”, en Ibidem, pp.6-9.

16 Pérez, "Gulf Dreams", p. 107.

17 Cherríe Moraga: Giving up the Ghost, en Heroes and Saints and Other Plays, pp.1-36, p. 8.
} 
generación, pero también que dicho modelo necesita reescribirse en el caso de las escritoras, puesto que la conclusión tradicional del "aprendizaje" de una protagonista femenina es la aceptación de su rol en la sociedad patriarcal. ${ }^{18}$ Esta reescritura del Bildungsroman, a menudo de fuerte base autobiográfica, ha sido llevada a cabo por un buen número de escritoras estadounidenses al menos desde los años sesenta, y forma parte en particular de la tradición literaria creada por mujeres de color estadounidenses, con la que enlazan estas autoras. La publicación en 1981 de la antología This Bridge Called My Back: Writings by Radical Women of Color, en cuya edición participaron Moraga y Anzaldúa, y que es de los primeros volúmenes que recoge la obra de autoras chicanas lesbianas, es el ejemplo más claro de este vínculo. Por otro lado, el énfasis en la adolescencia es igualmente propio de otra tradición específicamente homosexual, la de la "coming out story” o narrativa de salida del armario, que también actúa como modelo más o menos consciente.

En el caso que nos ocupa, el aprendizaje de las protagonistas consiste en el conocimiento de sí mismas, la aceptación de su identidad sexual y la búsqueda de su lugar en un mundo que las margina no sólo por ésta última, sino por su género y su "raza". Y tanto en la ficción como en los escritos de pensamiento, este conocimiento de sí mismas se origina en el cuerpo. La reivindicación del cuerpo y del conocimiento intuitivo, asociados tradicionalmente con lo femenino y relegados por ello a la categoría de "lo inferior" en el sistema de dualidades supuestamente complementarias que establece el patriarcado, ha sido uno de los elementos característicos del pensamiento feminista. ${ }^{19}$ Gloria Anzaldúa retoma esta idea al argumentar en Borderlands/La frontera que el cuerpo, lejos de ser ignorante, es sabio, y al criticar la dualidad cuerpo/espíritu, que considera especialmente propia del patriarcado cristiano occidental. ${ }^{20}$

Esta valoración del cuerpo y de lo intuitivo implica una serie de riesgos, como ya han hecho notar algunas pensadoras. ${ }^{21}$ La glorificación de la intuición y la emocionalidad de las mujeres que desarrolló el primer feminismo anglosajón corría el fuerte riesgo de reinscribir como innatos los atri-

\footnotetext{
18 Julián Olivares: "Entering The House on Mango Street", en John Maitino and David R. Peck (eds.): Teaching American Ethnic Literatures: Nineteen Essays, pp. 289-228, p. 212.

19 Véase, por ejemplo, Helène Cixous: Sorties: Out and Out: attacks/ways out/forays, selección en Douglas Tallack (ed.): Critical Theory: A Reader, pp. 200-211.

20 Anzaldúa, Borderlands/La frontera, p. 59.

21 Véase, por ejemplo, Monique Wittig: "The Straight Mind", en Douglas Tallack (ed): Critical Theory: A Reader, pp. 246-252.
} 
butos tradicionales de las mujeres y los hombres. De modo similar, la reivindicación del cuerpo llevada a cabo por la segunda ola del feminismo fue uno de los factores que contribuyó a la desafortunada vinculación entre la "liberación femenina" y la llamada "revolución [hetero]sexual", que distó mucho de ser verdaderamente liberadora para las mujeres, además de contribuir a un efecto parecido en lo que se refiere a la reinscripción de dualidades esencialistas. Cuando a estas dualidades (hombre: espíritu / mujer: cuerpo) se superponen las derivadas de la raza (raza blanca: espíritu / otras razas: cuerpo), los peligros se multiplican. Las consecuencias de estos errores, y el intento de las escritoras y críticas chicanas por solventarlos, se ponen de manifiesto de modo muy claro en uno de los ensayos publicados en el volumen conmemorativo This bridge we call home, en el que se celebran los veinte años de la publicación de This Bridge Called My Back. El ensayo describe la experiencia de cuatro profesoras que imparten conjuntamente un curso universitario titulado "US Latina Feminisms", e incide en la resistencia de sus alumnas a adquirir un bagaje intelectual a través del conocimiento y análisis de los textos teóricos sobre el tema, aunque estén escritos igualmente por mujeres de color. Llevadas por la reivindicación (igualmente de origen teórico, aunque ellas no lo sepan) de la experiencia directa (lo que las autoras denominan "testimonio") como única fuente válida de conocimiento, estas alumnas limitan de modo muy serio su capacidad de comprensión y de crítica del mundo. Como argumentan las autoras,

Theorizing is not a luxury for the use of those who remain outside the comprehensibility of white-supremacist-capitalist-patriarchal narratives. In fact, historically theorizing has proved vital for thriving despite white-supremacist-capitalist-patriarchal state agendas (...)

Viewing theory as something only white people do accommodates those state narratives that associate thinking with people of European descent and body with the rest of the world. ${ }^{22}$

En la misma línea, ya en la primera antología de lesbianas chicanas, Emma Pérez incluía un ensayo densamente teórico en el que reivindicaba la necesidad de emplear este tipo de conocimiento de modo crítico, ${ }^{23}$ y de hecho, como he explicado arriba, la producción de teoría ha ido paralela a la producción de ficción casi desde los primeros textos de estas autoras.

22 Sarah J. Cervenak et alt. (eds.): "Imagining Differently: The Politics of Listening in a Feminist Classroom", en Anzaldúa y Keating (eds.), This bridge, pp. 341-356, pp. 350, 351.

23 Emma Pérez: "Sexuality and Discourse: Notes from a Chicana Survivor", en Trujillo (ed.), Chicana Lesbians, pp. 159-184. 
Habiendo hecho todas estas salvedades en lo que se refiere a la teoría, lo cierto es que en los textos poéticos y de ficción de las escritoras chicanas que nos ocupan el conocimiento adquirido a través del cuerpo sí tiene un papel fundamental en la construcción de la identidad lésbica. En ellos abundan los momentos en los que la percepción a través de los sentidos (quizá con un énfasis particular en el olfato y el tacto, posiblemente los menos mediatizados por la palabra) desencadena una revelación para las protagonistas, narradoras o voces poéticas. Así, Gloria Anzaldúa evoca las sensaciones físicas de Andrea, la protagonista adolescente de "The Paisano is a Bird of Good Omen", cuando baila con otra muchacha:

"Ven. Baila conmigo". The López girl smiles, her teeth gleam as Andrea takes her into her arms and whirls her around the circle. Andrea s head is full of the music... The beat becomes her heart-beat, opening her, widening her diaphragm, her hips expanding. Only the tune exists and Belinda López. Throbbing. ${ }^{24}$

En "La Ofrenda", de Cherríe Moraga, se narra el acercamiento entre dos mujeres lesbianas, amigas desde la infancia, a través de un único encuentro sexual cargado de emoción y de significado. La descripción que hace la narradora del cuerpo de su amiga Tiny (Christina Morena), que se ha desnudado delante de ella en un arrebato para preguntarle a la narradora si la mujer de la que está enamorada podría desearla, ejemplifica perfectamente el aspecto positivo de la reivindicación de lo físico y lo real del cuerpo, en este caso frente a los estereotipos patriarcales y racializados de la belleza:

She's absolutely beautiful. Not magazine beautiful, but thirty-three years old and Mexican beautiful... Christina Morena desnuda sin a stitch on her body, and she looks like her mother and my mother, with legs like tree trunks and a panza that rolls round into her ombligo como pura miel. ${ }^{25}$

El olor y el tacto son los que desencadenan el encuentro sexual, que a su vez se transforma en una fuente de conocimiento al ser capaz de traspasar la barrera de la masculinidad y dureza asumidas de Tiny. Cuando ésta acepta que otra mujer le procure placer activamente, acaba con la fractura entre lo que Norma Alarcón denomina "male-like subjectivity and beha-

24 Gloria Anzaldúa: “The Paisano is a Bird of Good Omen”, en Alma Gómez et alt. (eds.): Cuentos: Stories by Latinas, pp. 153-175, p. 171.

25 Cherríe Moraga, "La Ofrenda", p. 6. 
vior" y su "literal female body", la fractura entre su ser mujer y su deseo por otras mujeres, ${ }^{26}$ obteniendo un conocimiento de sí misma que es una fuente de poder.

Si el cuerpo es lo que lleva al reconocimiento y la aceptación de la identidad lésbica, también es el cuerpo el que rechaza vivamente lo que Adrienne Rich denominara heterosexualidad obligatoria. ${ }^{27}$ Con la excepción de la novelista y poeta Ana Castillo, que se declara bisexual, pero que ha participado en algunas de las antologías citadas con análisis muy lúcidos de la identidad lésbica chicana, ${ }^{28}$ la práctica totalidad de las escritoras citadas denuncian la presión externa para mantener relaciones heterosexuales en contra del conocimiento que una vez más les proporciona su cuerpo. Un ejemplo claro puede encontrarse en la expresión de repugnancia de Sor Juana Inés de la Cruz en la novela de Alicia Gaspar de Alba, que concreta la "total negación que tenía para el matrimonio" manifestada por la Sor Juana real:

Juana Inés cerró los ojos e imaginó las manos de un hombre sobre su cuerpo, sus labios y barba sobre su rostro, su vientre cargado de hijos, su mente arrugada como una pasa.

— ¡Oh, no, padre! — imploró al tiempo que se aferraba a las amplias mangas de su sotana-. Os ruego que no me obliguéis a contraer matrimonio. ¡Por favor, padre!29

Es el mismo rechazo muy físico que la comediante Mónica Palacios incluye como parte de su monólogo "Tomboy", con la diferencia obvia de que ella se dirige a un público que se identifica, o al menos puede simpatizar, con la expresión de este sentimiento:

Oh god and the sperm. And there was always so much of it.

Where did it come from? Sperm on tap!

I couldn't handle it.

26 Norma Alarcón: "Making Familia from Scratch: Split Subjectivities in the Work of Helena María Viramontes and Cherríe Moraga", en María Herrera-Sobek and Helena María Viramontes (eds.): Chicana Creativity and Criticism: Charting New Frontiers in American Literature, pp. 147-159, p. 155.

27 Adrienne Rich: "Compulsory Heterosexuality and Lesbian Existence", Signs vol 5, n. 4 , pp. 631-660.

28 Ana Castillo: "La Macha: Towards a Beautiful New Self", en Trujillo (ed.), Chicana Lesbians, pp. 24-47; Martha A. Navarro: "Interview with Ana Castillo", en Ibidem, pp.113-132.

29 Alicia Gaspar de Alba: El segundo sueño, p. 63. Sor Juana justifica su elección de la vida conventual basándose precisamente en esta "total negación": F.L. Cardona: "Estudio preliminar", Sor Juana Inés de la Cruz: Poesía Lírica y El divino Narciso, p. 8. 


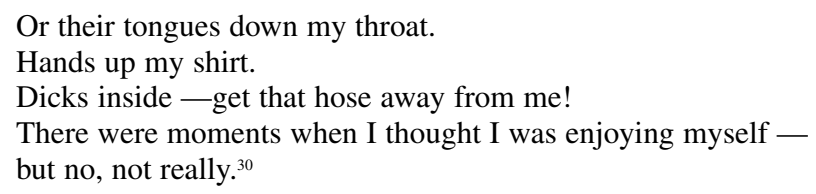

La misma existencia de una "stand up comedian" lesbiana y chicana demuestra las transformaciones que se han ido produciendo en ciertos ambientes de la sociedad (en especial en el ámbito urbano y universitario), transformaciones que hacen posible para algunas mujeres vivir abiertamente una existencia lésbica sin fisuras, donde esa heterosexualidad obligatoria apenas parece afectar ya a sus vidas. Es el mundo que aparece en relatos como "They're Just Silly Rabbits" (1987), de Gaspar de Alba, o en "Personality Fabulosa" (1995), de la propia Palacios. ${ }^{31}$

No obstante, los relatos de este tipo sin duda continúan siendo minoritarios y un buen número de obras reflejan la amargura y el sufrimiento que genera en la existencia de las protagonistas la presión del patriarcado, a veces en su expresión más brutal. En la ya citada Giving up the Ghost, de Moraga, Corky describe en un monólogo estremecedor la violación que sufrió a la edad de doce años y el efecto devastador de este ataque sobre su identidad y su autoestima: "He made me a hole!" se lamenta Corky. Su contrapartida adulta, Marisa, razona con sarcasmo que la experiencia "makes you more aware than ever that you are one hunerd percent female, just in case you had any doubts. One hunerd percent female whether you act it... or like it... or not". ${ }^{32}$ Otros textos, como "Pesadilla", también de Moraga (1983), o "Dreams of Violence", de Naomi Littlebear (1981), relatan agresiones violentas sufridas por parejas de mujeres. ${ }^{33}$ Se denuncia esta violencia sin caer nunca en el tópico de que el lesbianismo es de alguna forma el resultado de las agresiones masculinas, un tópico que Ana Castillo, en "La Macha", desmonta con cierto humor al afirmar que si el lesbianismo fuera meramente una reacción a la dominación masculina, la inmensa p. 308 .

30 Mónica Palacios: “Tomboy”, en Carla Trujillo (ed.): Living Chicana Theory, pp. 305-309,

31 Alicia Gaspar de Alba: "They're just Silly Rabbits," en Sergio D. Elizondo y Armando Armengol (ed.), Palabra nueva: Cuentos chicanos II, pp. 107-114. Mónica Palacios: "Personality Fabulosa”, en Lillian Castillo-Speed (ed.): Latina: Women's Voices from the Borderlands, pp. 200-207.

32 Moraga, Giving up the Ghost, pp. 25, 29.

33 Cherríe Moraga: "Pesadilla", en Living in the War Years: Lo que nunca pasó por sus labios, pp. 36-43; Naomi Littlebear Morena: "Dreams of Violence", en Anzaldúa y Moraga (eds.), This Bridge Called my Back, pp. 16-17. 
mayoría de las mujeres del mundo se habrían vuelto lesbianas. ${ }^{34}$ Las escritoras estudiadas son también muy explícitas a la hora de dar voz a los sentimientos de dolor y de traición que experimentan sus personajes cuando sienten que el poder patriarcal les arrebata a las mujeres que aman y las sitúa en una posición de inferioridad sexual y existencial frente a los hombres. Al hacerlo, con una autoridad que brota tanto del "testimonio" del sentimiento personal como de la "teoría" lesbiana y feminista, confrontan algunos de los fantasmas más arraigados de la existencia lésbica, y se desmarcan de esa nueva escritura "post-lésbico-feminista" influenciada por las teorías queer con su reivindicación de la indefinición de género, la experimentación bisexual y las sexualidades alternativas. Los tonos en los que se expresan estos sentimientos abarcan de nuevo desde la ironía más o menos humorística hasta el dolor más crudo. E.D. Hernández lo narra del primer modo:

After several months of besitos, kissitos and a series of ritual false promises, your querida indica que también le gustan los hombres... bueno, pues at the risk of sounding macho, I kept quiet after the revelation and sometime after became secretly repulsed. No sé por qué, pero me dió un asco and the luscious little love affair was over. $^{35}$

En cambio Moraga, a través del personaje de Marisa, lo expresa de forma concisa y desoladora: "The women I have loved the most have always loved the man more than me, even in their hatred of him" ${ }^{36}$

En suma, las obras mencionadas construyen una identidad lésbica chicana con unos rasgos bastante específicos. Es una identidad que parte de la reivindicación que supone nombrarse a sí misma; que no cuestiona sus orígenes, sino que se admite como innata; que se revela a través de la conciencia del cuerpo, tanto en su deseo del cuerpo femenino como en su rechazo de la heterosexualidad; y que en estos textos combina el conocimiento derivado de la experiencia con el apoyo derivado de la teoría feminista. En todo esto, y dejando de lado por el momento los condicionantes más específicamente chicanos (raciales y culturales), la identidad que se refleja en la mayoría de los textos coincide a grandes rasgos con la concepción de la identidad lésbica defendida por el feminismo lésbico radical, más que con

34 Ana Castillo: "La Macha", p. 35.

35 E.D. Hernández: "Discussion, Discourse and Direction: The Dilemmas of a Chicana Lesbian”, en Trujillo (ed.), Chicana Lesbians, pp. 138-140, p. 139.

36 Moraga, Giving up the Ghost, p. 14. 
las sexualidades alternativas de la teoría queer. Hay no obstante un elemento "heterodoxo" que merece analizarse: se trata de la frecuente aparición de personajes, o parejas, que podrían incluirse dentro del juego de roles conocido como butch/femme: la "marimacha" de rasgos y comportamientos "masculinos" como la versión chicana de la butch, frente a la mujer lesbiana sensual y "femenina", la femme chicana. El feminismo lésbico de los años 70 criticó este patrón de relación lesbiana en tanto que consideraba que tendía a reproducir precisamente el modelo heterosexual de desigualdad que el feminismo buscaba abolir. En los años 80 se reabrió el debate, a veces desde una reivindicación peligrosa de la erotización de la desigualdad, a veces desde la perspectiva queer del género como construcción, y otras desde una arraigada percepción subjetiva de la propia identidad. ${ }^{37}$

Conscientes de la presencia de mujeres que se identifican con estos roles dentro de la cultura chicana, las autoras describen y analizan dichas identidades, revelando una complejidad que va mucho más allá de la supuesta reproducción de roles heterosexuales. Yolanda Chávez Leyva hace notar que en el contexto chicano, y en particular antes de la concienciación feminista, los modelos más visibles de identidad lésbica fueron precisamente las "marimachas", las mujeres butch que con su ropa y sus modales "masculinos" rompían visualmente el silencio de la comunidad latina. ${ }^{38}$ Ana Castillo, por su parte, relaciona la persistencia de este modelo con la fuerza de la presión patriarcal dentro de la cultura chicana, señala los condicionantes económicos y sociales específicos que impiden a muchas mujeres vivir su identidad de otra manera, y sobre todo insiste en que cualquier juego de poder dentro de las parejas de roles no es equivalente al modelo heterosexual, ya que en el caso de las lesbianas, no hay ninguna sanción externa que respalde la dominación que se ejerce dentro de la relación. ${ }^{39}$ Gloria Anzaldúa lo conecta con el concepto popular mexicano de "mita y mita", según el cual se concibe a la lesbiana "marimacha" como un ser intermedio entre lo masculino y lo femenino. ${ }^{40}$

Los personajes de este tipo reflejan una realidad social, pero también contribuyen a deshacer las simplificaciones sobre los roles, en especial la

37 Sheila Jeffreys (La herejía lesbiana), Lillian Faderman (Surpassing the Love of Men) y Carole Vance (ed.) (El placer y el peligro), dan cuenta de estos debates ideológicos.

38 Yolanda Chávez Leyva: "Listening to the Silences in Latina/Chicana Lesbian History", en Trujillo, Living Chicana Theory, pp. 429-434, p. 434.

39 Ana Castillo, "La Macha", p. 36.

40 Anzaldúa, Borderlands/La frontera, p. 41. 
idea popular según la cual se limitan a una simple reproducción caricaturesca de la heterosexualidad. La ternura y vulnerabilidad de personajes como Corky o Tiny, en las obras ya mencionadas de Moraga, o la ingenuidad de la butch Gloria, de los relatos de Rocky Gámez ${ }^{41}$ — características que dentro del patriarcado se asumen como "femeninas"- muestran una realidad mucho más compleja. En este sentido, resulta esclarecedor el ensayo autobiográfico de Cherríe Moraga sobre su experiencia como madre lesbiana. Moraga hace notar la paradoja de que el hecho de haber asumido ella —la integrante más butch, más "marimacha" de la pareja— la maternidad biológica la hace objeto de una aceptación a la que no estaba acostumbrada, mientras que su pareja femme, más "femenina", de repente se ve convertida en "the dyke in the matter", algo que resulta duro para una mujer que "has always passed effortlessly - that is, until she opens her mouth and the lesbian feminist rolls out without restraint". ${ }^{42}$ Las expectativas sobre la "masculinidad" y la "feminidad" de los respectivos roles se rompen tanto por la maternidad biológica —el rol femenino por excelencia - de la parte supuestamente masculina como por la militancia radical de la femme, lo que sugiere una vez más que los roles nada tienen que ver con su versión caricaturesca.

En cuanto a esto, en el ya citado volumen conmemorativo This bridge we call home el ensayo de Max Wolf Valerio confirma esta idea desde la perspectiva de su experiencia como transexual de mujer a hombre. Valerio, que había participado en la primera antología con un trabajo - escrito antes de su cambio de sexo- en el que hablaba de sus problemas infantiles con la identidad femenina, que le habían llevado a reconocerse como lesbiana butch, ${ }^{43}$ explica en la segunda cómo esta identificación había llegado a no corresponderse con sus sentimientos sobre sí mismo, basados una vez más en el cuerpo. Lo que me interesa destacar aquí es que Valerio manifiesta lo siguiente:

What has not changed is, essentially, that lesbianism is a world of femaleness, even if some lesbian women are actively questioning their gender identity and hesitate to call themselves "women".

\footnotetext{
41 Rocky Gámez: "From The Gloria Stories", en Alma Gómez et alt. (eds.), Cuentos: Stories by Latinas, pp. 138-146. p. 184.

42 Cherríe Moraga: "Free at Last", en Trujillo (ed.), Living Chicana Theory, pp.166-188,

43 Max Wolf Valerio: "It's in My Blood, My Face - My Mother's Voice, The Way I Sweat", en Anzaldúa y Moraga (ed.), This Bridge Called My Back, pp. 41-46.
} 
Esta afirmación del lesbianismo como "a world of femaleness" marca de nuevo una separación clara entre la identidad butch — que se mantiene dentro de ese mundo de mujeres - y la identidad masculina. Por otra parte, Valerio rechaza también las ambivalencias de género de la ideología queer, al menos en lo que se refiere a su propia experiencia de sí mismo como hombre heterosexual "relatively gender-role congruent". ${ }^{44}$ Es cierto que, más recientemente, trabajos como el vídeo "boi haircut" de Alma López (que documenta la conversación de tres lesbianas de color sobre el significado de sus cortes de pelo "a lo chico") sí se enmarcan en la conceptualización casi postmoderna de la "performance" deliberada de una cierta identidad, en la representación de un género elegido, pero sin renunciar a la identificación como mujeres y como lesbianas. ${ }^{45}$

\section{La identidad como traición}

Not me sold out my people but they me.

Gloria Anzaldúa ${ }^{46}$

Un aspecto específico y central de la literatura lésbica chicana es su necesidad de confrontar la acusación de que la identidad lésbica es incompatible con la identidad chicana, hasta el punto de constituir una traición a la misma. Todavía en 2002, y aun consciente de que está repitiendo algo que ha llegado a ser un cliché, Renée M. Martínez recuerda vivamente su miedo a la imposibilidad de reconciliar ambas identidades ("being a chicana and a lesbian, my parents' daughter and a lesbian, alive and a lesbian"), su miedo a que ser lesbiana "would sever me from everything that counted in my life: Homosexuality, the ultimate betrayal of my Mexican heritage, was only for white people". ${ }^{47}$

Hay una larga tradición de asociar la homosexualidad con la traición a la patria, cuyas consecuencias más terribles se manifiestan en la brutal

44 Max Wolf Valerio: “ 'Now that You are a White Man': Changing Sex in a Postmodern World-Being, Becoming, and Borders", Anzaldúa y Keating (eds.), This bridge we call home, pp. 239-254, p. 242, p. 252.

45 Alma López, "boi hair," http://www.almalopez.net/video/boi.html, (11-11-2009)

46 Anzaldúa, Borderlands/La Frontera, p. 44.

47 Renée M. Martínez: "Del puente al arco iris: transformando de guerrera a mujer de la paz. From Bridge to Rainbow: Transforming from Warrior to Woman of Peace," Anzaldúa y Keating (eds.), This bridge we call home, pp. 42-50, p. 45. 
represión de los homosexuales en los regímenes totalitarios de uno y otro signo. Si bien esta idea se ceba en los homosexuales varones, las lesbianas, en tanto que homosexuales, también pueden sufrirla. Pero, adicionalmente, las lesbianas son tildadas de antipatrióticas y decadentes simplemente como mujeres críticas con el sistema patriarcal, una acusación que comparten con las feministas. Centrándonos en lo más específico, la feminista lesbiana blanca estadounidense Sheila Jeffreys, entre muchas otras pensadoras, analiza las razones por las que la identidad lésbica se construye como "la herejía lesbiana" que da título a su libro. Jeffreys afirma que las lesbianas, más aún que las feministas heterosexuales, expresan su negativa radical a adoptar el rol que se espera de las mujeres en el patriarcado. El hecho de no mantener relaciones heterosexuales implica "retirar de la circulación" cuerpos de mujer que en teoría son propiedad de los varones del grupo, tanto para la sexualidad como para la reproducción. Más allá de las prestaciones sexuales y reproductivas, las lesbianas retiran de la circulación heterosexual una serie de "servicios" de apoyo práctico, emocional y psicológico que en principio los varones reciben de las mujeres. Por añadidura, las lesbianas escapan a las definiciones clásicas de la identidad femenina, que siempre se hacen con respecto a la relación con un varón (novia de, esposa de). Finalmente, al mantener relaciones sexuales con otras mujeres, las lesbianas se apropian de ese derecho al acceso al cuerpo de las féminas que se considera propio de los varones, y también destinan a otras mujeres la energía que se empleaba en los "servicios de apoyo" al varón anteriormente mencionados. ${ }^{48}$

Al formar parte de un grupo cultural no hegemónico, minoritario, la identidad de las lesbianas chicanas se ve aún más en términos de traición a la comunidad de origen y a la familia: a todos los factores anteriores se le añaden condicionantes específicos que agravan la "herejía". En primer lugar, las identidades de los grupos minoritarios a menudo se establecen marcando contrastes con el grupo dominante, y para ello se refuerzan las identidades "tradicionales" de género dentro del grupo minoritario. En el caso de los chicanos varones, la masculinidad "latina" se define como violenta y agresivamente heterosexual frente a la (supuesta) "domesticación" y "feminización" de los varones anglosajones. Paralelamente, se atribuye a las mujeres chicanas una feminidad igualmente más marcada, con una división tajante entre "buenas" y "malas" mujeres en términos de sexualidad, y

48 Sheila Jeffreys: La herejía lesbiana. 
un gran énfasis en la obediencia y la sumisión, en la aceptación del sufrimiento. Como denuncia Carla Trujillo, "our toleration of unjust behavior from men, the church, the established order, is considered an attribute (...) Martyrdom, the cloth of denial, transposes itself into a gown of cultural beauty". ${ }^{49}$

Gloria Anzaldúa hace notar que en el ámbito chicano las mujeres son empleadas como depositarias de la tradición: "The culture expects women to show greater acceptance of, and commitment to, the value system than men", ${ }^{50}$ una idea que Celia Amorós desarrolla también en referencia a otras comunidades. Según Amorós, las mujeres, que se consideran esencialmente invariables, a-históricas y propiedad de la comunidad de varones, se utilizan para encarnar la continuidad y la tradición de una cultura minoritaria, y lo ejemplifica con el caso de los inmigrantes musulmanes en Europa: mientras que los hombres pueden evolucionar, como se manifiesta en el uso de ropa "occidental", las mujeres deben servir como símbolo de la persistencia de los valores tradicionales y manifestarlo en su vestimenta (en especial, mediante el velo islámico) ${ }^{51}$

Por añadidura, dentro de los grupos minoritarios que se sienten amenazados, las mujeres se consideran especialmente valiosas como instrumentos de reproducción para asegurar la continuidad física de la comunidad. En un estudio sobre la homosexualidad dentro de la comunidad judía, Alan Unterman señala que, al imperativo religioso que impone el matrimonio heterosexual y la reproducción, se suma el imperativo histórico de utilizar el crecimiento demográfico como arma de supervivencia; en este sentido, Carla Trujillo se refiere a la creencia errónea dentro de la comunidad chicana de que "the only way to uplift the species is to propagate it" ${ }^{52}$ Con todo lo anterior, resulta claro que nada más como mujeres feministas, como mujeres rebeldes, que se niegan a adoptar esos roles femeninos, a asumir su papel de depositarias de la tradición y a procrear para beneficio de la

49 Carla Trujillo: "Chicana Lesbians: Fear and Loathing in the Chicano Community", en Alma M. García (ed.): Chicana Feminist Thought, pp. 281-287, p. 283.

50 Anzaldúa, Borderlands/La Frontera, p. 39.

51 Celia Amorós: "Feminismo, multiculturalismo e Ilustración," conferencia pronunciada en la Universidad de Sevilla, 2002. Sin negar en absoluto el espíritu rebelde de la pintora mexicana, Frida Kahlo de algún modo sigue este patrón al adoptar la vestimenta tradicional mexicana, al contrario que su esposo Diego Rivera.

52 Alan Unterman: "Judaism and Homosexuality: Some Orthodox Perspectives", en Jonathan Mangonet (ed.) Jewish Explorations of Sexuality, pp. 67-74; Trujillo (ed.), "Chicana Lesbians: Fear and Loathing," en García, Chicana Feminist Thought, p. 284. 
demografía de la comunidad chicana, las lesbianas chicanas pueden ser consideradas traidoras a [los varones de] su raza.

Todas las ideas anteriores tienen un cariz absolutamente conservador y evocan los discursos patrióticos del nacionalsocialismo, por poner sólo un ejemplo; por tanto, sería de esperar que hubieran cambiado en el contexto de los movimientos civiles de los años 70, en los que se enmarca el movimiento chicano. El pensamiento revolucionario de origen marxista que animó muchas de las reivindicaciones de estos grupos, y que debería haber implicado una crítica a la tradición, una aceptación del análisis de la sumisión de las mujeres dentro del capitalismo y una visión internacionalista, distó mucho de llevarlo a cabo. Como en el contexto de muchos movimientos similares (un ejemplo español es el de la rebelión antifranquista), el argumento empleado fue que el socialismo, la lucha de clases, era el primer objetivo a perseguir, y que una vez obtenida esa victoria se podría prestar atención a la situación de las mujeres, que por añadidura evolucionaría como resultado de los cambios sociales. Como resume Emma Pérez, "The global socialist movements in the early twentieth century in Europe, Russia, China and the Americas inevitably found women forced to place gender issues as secondary to male-defined arguments". El movimiento chicano de los años 70 no rompió esta dinámica, a pesar de los esfuerzos de muchas mujeres que participaron en él, y Pérez se lamenta: "We are tired of debating the same questions that plagued Alexandra Kollontai in Russia and Hermila Galindo in Yucatán in 1917". ${ }^{33}$ Resulta fácil comprender que, en este contexto, las reivindicaciones de las chicanas lesbianas (concebidas como un grupo aún más minoritario y más contrarrevolucionario que las feministas heterosexuales) fueran no sólo desatendidas, sino violentamente censuradas. En palabras de Catriona Rueda Esquibel:

It's crucial to recognize that the first articulations of feminist goals and struggles within the Chicano/a movement were marked by a homophobic backlash in which all Chicana feminists were subject to lesbian-baiting, at both personal and professional levels. $^{54}$

La idea del feminismo (y mucho más del lesbianismo) como un implante "blanco", una contaminación del mundo anglosajón de efectos enormemente destructivos para la vitalidad del movimiento chicano, se

53 Emma Pérez, "Sexuality and Discourse: Notes from a Chicana Survivor," en Trujillo (ed.), Chicana Lesbians, pp. 159-184, p. 159.

54 Rueda Esquibel, With her Machete in her Hand, p. 3. 
repitió hasta la saciedad, y a ella hacen referencia una multitud de textos teóricos recogidos en antologías como Chicana Feminist Thought: The Basic Historical Writings o Living Chicana Theory. Autoras lesbianas como Gloria Anzaldúa, Emma Pérez, Carla Trujillo, Ana Castillo, Natashia López, para mencionar sólo unas cuantas, emplean el término y/o el concepto de traición, intentando rebelarse contra él. Uno de los análisis más brillantes y exhaustivos es el de Cherríe Moraga en su ensayo fuertemente autobiográfico "A long line of Vendidas," de 1983:

The woman who defies her role ... is purported to be a "traitor to her race" by contributing to the "genocide" of her people ... In short, even if the defiant woman is not a lesbian, she is purported to be one; for, like the lesbian in the Chicano imagination, she is una Malinchista. Like the Malinche of Mexican history, she is corrupted by foreign influences which threaten to destroy her people.

En el caso específico del lesbianismo, continúa Moraga,

Lesbianism can be construed by the race then as the Chicana being used by the white man, even if the man never lays a hand on her. The choice is never seen as her own. Homosexuality is his disease with which he sinisterly infects Third World people, men and women alike..$^{55}$

Frente a todo esto, la actitud de muchas de estas mujeres desafiantes, lesbianas o no, fue la de marcar claramente los límites de su lealtad a la comunidad chicana y a sus "carnales" varones. La formulación puede ser más o menos sofisticada, pero la cantautora lesbiana Naomi Littlebear Morena lo resume de modo contundente: "i agree, me and my 'brother' both been screwed by the system, but when he starts screwin' me he is the system". 56

Resultó, en cambio, más problemático librarse de la priorización de la raza frente al género en lo que se refiere al cuestionamiento del feminismo "blanco", de raíz anglosajona. Como ya se apuntó en el debate sobre el uso de la "teoría", persiste en la comunidad académica chicana y en el activismo de base el debate sobre las limitaciones del feminismo blanco y la necesidad de adoptar un feminismo específico, que tenga en cuenta la experiencia vital de las mujeres de color con todos sus condicionantes de raza, clase, lengua, etc. Se acusaba asimismo al feminismo "blanco" de no

55 Cherríe Moraga, “A Long Line of Vendidas", en Living in the War Years, pp. 90-144, p. 114.

56 Naomi Littlebear Morena: "Coming out Queer and Brown”, en Sara-Lucia Hoagland y Julia Penelope (eds.): For Lesbians Only, pp. 346-347, p. 347. 
enfrentarse con su propio racismo y con las implicaciones de las mujeres blancas en las estructuras de poder, así como de un exceso de intelectualismo y abstracción. ${ }^{57}$ Autoras como Sonia Saldívar-Hull, Gloria Anzaldúa, Norma Alarcón, Cherríe Moraga y Emma Pérez abordaron, desde posiciones distintas, este debate, especialmente en la década de los 90, y en varios textos de la antología de 2002, This bridge we call home, se muestra su persistencia. No tengo espacio aquí para entrar en detalle en esta discusión, pero desde mi propio posicionamiento, que enfatizaría más las similitudes que las diferencias entre feminismos, quisiera subrayar que en este debate también entra, a mi parecer, ese miedo subconsciente a traicionar a la comunidad de raza. De nuevo, me parecen muy pertinentes en este sentido las observaciones de Cherríe Moraga, que hace notar que, frente a la duda de algunas feministas chicanas sobre si es deseable o no emplear fuentes escritas por mujeres blancas, los fundadores varones del movimiento chicano no veían inconveniente alguno en emplear los análisis de un pensador blanco como Marx. Por añadidura, y quizá de modo aún más pertinente, Moraga critica la tendencia a olvidar que las feministas blancas (y en particular las lesbianas) se están rebelando también contra su propia cultura, "challenging the authority of [their] white father" y por tanto pueden ser consideradas aliadas. ${ }^{58}$

Sin embargo, romper el miedo subconsciente a la acusación de traición no es fácil, y quizá es por esto por lo que resulta tan crucial para todas estas autoras el intento de mantener los vínculos con la comunidad chicana, y en especial con la familia, a pesar de su conciencia de la absoluta necesidad de ciertas formas de separación, de crítica y de rechazo frente a una cultura que les puede llevar a sentir, como Gloria Anzaldúa, que no tienen existencia como individuos: "No self, / Only race vecindad familia" ${ }^{59}$

La vinculación, y los desencuentros, con la familia de origen es un tema fundamental y muy doloroso no sólo en la obra de las lesbianas chicanas, sino para las lesbianas y homosexuales en general. Debido a los factores específicos del contexto latino y católico, quizá en el caso que nos ocupa haya un énfasis añadido en lo que significa el vínculo familiar. Ana Castillo sugiere que dentro de la sociedad estadounidense muchas mujeres

57 Véase, por ejemplo, Norma Alarcón: "The Theoretical Subjects of This Bridge Called my Back and Anglo-American Feminism", pp. 28-39, o Sonia Saldívar-Hull, "Feminism on the Border: From Gender Politics to Geopolitics”, pp. 202-220, ambos en Héctor Calderón y José David Saldívar (eds.): Criticism in the Borderlands: Studies in Chicano Culture.

58 Cherríe Moraga, Loving in the War Years, p. 106.

59 Anzaldúa, Borderlands/La Frontera, p. 195. 
chicanas sienten que el único sitio donde tienen un lugar es dentro de la familia y que el riesgo de perder este lugar, así como el amor y el respeto que se tiene dentro de la familia se ve como un precio demasiado alto. ${ }^{60}$ Las relaciones de la familia de origen con los hijos e hijas homosexuales en EEUU han sido objeto de análisis, muy recientemente, por la novelista y activista Sarah Schulman, de origen judío neoyorkino, que ha estudiado en profundidad la familia como fuente de homofobia. ${ }^{61}$ Algunas de las conclusiones de estos estudios parecen muy aplicables a cualquier cultura y remiten de nuevo a la idea de traición, una traición que consiste en no adecuarse a las expectativas de futuro que los padres y madres se formulan con respecto a los hijos. De hecho, Erik F. Strommen y Bryan E. Robinson van más allá cuando afirman que la revelación de la identidad homosexual de una hija o un hijo produce una serie de reacciones comparables al proceso de duelo por la pérdida de un ser querido, ya que los padres tienen que asimilar que las esperanzas que tenían puestas en ellos no se van a cumplir. ${ }^{62}$ A esta traición generalizada se suman las concretas, como la que se refleja, por ejemplo, en las palabras de Martha Barrera cuando se lamenta de que su padre la llama Malinche por emparejarse con una mujer blanca en lugar de con un hombre mexicano. ${ }^{63}$

Junto con Gloria Anzaldúa, Cherríe Moraga es quizá la autora que más haya profundizado en las complejas relaciones entre familia, raza e identidad lésbica. Hija de padre "anglo" y de madre mexicana, en Loving in the War Years intenta explicar su propia identidad sexual remitiéndose tanto al complicado e intenso amor que recibe de su madre - que condiciona su propio amor por las mujeres, pero que siempre está amenazado por la preferencia no cuestionada de su madre por el hermano de Cherríecomo al distanciamiento y la posible ambigüedad sexual de su padre "anglo". Martha Barrera, sin embargo, hace notar que Moraga encuentra imposible compartir su escritura con sus padres y hace referencia al dolor que produce esta falta de comunicación, reflejando una situación común

60 Martha A. Navarro: "Interview with Ana Castillo", en Trujillo, Chicana Lesbians, pp. 113132, p. 120.

61 Sarah Schulman: Ties that Bind: Familial Homophobia and its Consequences, New York, New Press, 2009. Una recopilación de estudios anterior, pero aún útil, es la Frederick W. Bozett (ed.): Homosexuality and the Family.

62 Bryan E. Robinson et alt.: "Response of Parents to Learning that Their Child Is Homosexual and Concern over AIDS: A National Study”, p. 60, pp. 59-80; Erik F. Strommen: “'You're a What?': Family Member Reactions to the Disclosure of Homosexuality," p. 42, pp. 37-58, ambos en Bozett (ed.), Homosexuality and the Family.

63 Martha Barrera, "Café con leche", en Trujillo (ed.), Chicana Lesbians, pp. 80-83, p.80. 
entre escritoras/hijas lesbianas: "When you care about something so much that it is a part of your soul, and you can't share it with your parents because they don't/won't can't understand, how can you not [sic] help but feel alienated from them?"

Frente a este aislamiento se alza la denuncia que Anzaldúa expresa en las palabras que sirven de epígrafe a este apartado: la acusación de que la traición no la han llevado a cabo las hijas lesbianas, sino la familia y la comunidad de origen, que las han repudiado y abandonado. En su estudio de la homofobia familiar, Schulman insiste en la injusticia de los castigos a los que la familia heteropatriarcal somete a sus miembros homosexuales:

Because of the twisted nature of dominant behavior, gay people are being punished within the family structure even though we have not done anything wrong. And this punishment has dramatic consequences on both our social experiences, and our most trusting, loving sexual relationships. ${ }^{64}$

Se alza también el reconocimiento del valor de las nuevas familias que las autoras o sus personajes forman con sus parejas femeninas (y en algunos casos, con sus hijas e hijos), una familia que se extiende a la unión con otras mujeres lesbianas. Martha Barrera afirma: "We are just as valid a familia as we would be if she were a brown man who I married in the Catholic Church". ${ }^{65}$ No obstante, recurre al deseo de reconciliación con la familia de origen y este acontecimiento raro pero no imposible encuentra una expresión tierna y humorística en el breve poema de Juanita M. Sánchez, "Paso a paso":

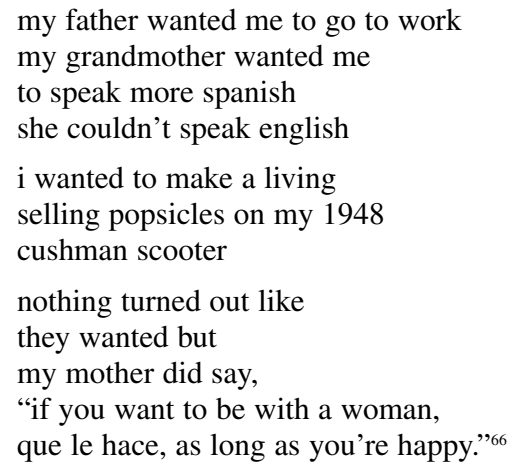


Por último, es necesario detenerse en uno de los medios que emplean estas escritoras y artistas para restablecer una vinculación con su comunidad, una vez confrontadas las acusaciones y las diferencias. Se trata de la reapropiación de mitos chicanos para expresar la identidad lésbica. Lógicamente, esta táctica no pretende, ni logra, evitar la virulenta reacción lesbófoba de la voz oficial de la comunidad chicana: en el mundo del arte, las imágenes "sacrílegas" de la Virgen de Guadalupe realizadas por Alma López fueron brutalmente denostadas y ella tachada de artista decadente en La voz de Aztlán. ${ }^{67}$ Se trata más bien de llevar a cabo la tarea que plantea Gloria Anzaldúa:

Myths and fictions create reality, and these myths and fictions are used against women and against certain races to control, regulate and manipulate us. I'm rewriting the myths, using the myths back against the oppressors...

There are certain myths - the stories of Coatlicue, la Llorona, la Chingada, la Virgen de Guadalupe, and Coyolxauhqui, the moon goddess - that I associate with women. I want to take these figures and rewrite their stories. The figures we're given have been written from the male patriarchal perspective. ${ }^{68}$

Lógicamente, esta tarea se enmarca en el contexto más amplio de la crítica y literatura feministas, y en el entorno chicano escritoras heterosexuales también han reescrito y analizado algunos de los mitos citados; la diferencia es el objetivo de darles una relectura que dé cabida a la experiencia lesbiana. En la obra de Anzaldúa, la reescritura de mitos llega al punto de conformar una especie de lenguaje propio, en el que los distintos estados del viaje espiritual de transformación que propone la autora se asocian con distintas figuras de la mitología náhualt: así, el descenso a los aspectos más temibles de la propia psique se asocia con la diosa Coatlicue, creadora y destructora, y la reconstrucción de la identidad con la recuperación de los fragmentos mutilados del cuerpo de la diosa Coyolxauhqui, descuartizada por su hermano en esta mitología. ${ }^{69}$ Por su parte, Cherríe Moraga ha combinado mitologías clásicas y mexicanas en su obra The Hungry Woman: A Mexican Medea (1995), y su empleo de la figura de Malinche

\footnotetext{
67 Editorial, 23 de abril de 2001. http://www.almalopez.net/ORnews/010423az.html. (11-112009).

68 Gloria Anzaldúa: “An Interview with Debbie Blake and Carmen Abrego (1994)”, en Ana Louise Keating (ed.): Interviews / Entrevistas, pp. 221-233, p. 219.

69 Este proceso empieza en 1987 en Borderlands/la Frontera y, debido a la prematura muerte de la autora, culmina en 2002 con su artículo "now let us shift ... the path of conocimiento ... inner work, public acts", en Anzaldúa y Keating (ed.), This bridge we call home, pp. 540-578.
} 
en "A Long Line of Vendidas", como hemos visto, le permite analizar vívidamente el concepto de traición. ${ }^{70}$

Con un tono mucho más ligero, Mónica Palacios parodia otro mito, la trágica historia de la Llorona - existen varias versiones, pero la más común es que se trata del espíritu de una desgraciada mujer indígena que asesinó a sus hijos, habidos con un caballero español, cuando éste se casó con una dama española - en el relato "La Llorona Loca: The Other Side". En él, presenta en un contexto arquetípicamente mexicano la relación entre la bella Caliente y la poderosa Stranger, que aparece a caballo un día para llevarse a Caliente, la mujer más deseada del pueblo. Más tarde, cuando la Stranger confiesa que está engañando a Caliente con otra mujer, Caliente la ahoga en un arrebato de celos. La Llorona Loca del relato es el fantasma de la infeliz Caliente, que lamenta su crimen junto al río. ${ }^{71}$

Mucho más seria y exhaustiva es la reinterpretación y reivindicación lésbica de la figura de Sor Juana Inés de la Cruz que lleva a cabo la escritora Alicia Gaspar de Alba, ya citada. La religiosa, con su origen mestizo, su reivindicación de los derechos espirituales e intelectuales de la mujer y sus poemas de amor a la virreina de México, su protectora, se presta especialmente a esta tarea, y los escritos de Gaspar de Alba la abordan mediante el empleo de diversas formas literarias que culminan en su brillante novela histórica Sor Juana's Second Dream. Uno de los textos más complejos, que ejemplifica perfectamente la definición de "fiction/theory" es el titulado "The Politics of Location of the Tenth Muse of America", en el que utiliza el formato de una falsa entrevista en la que extrae las respuestas de Sor Juana de sus auténticos escritos para situarla a través de ellas como "a symbolic foremother of Chicana lesbian feminism". ${ }^{72}$

En conclusión, las escritoras chicanas lesbianas emplean la literatura, como ya dijimos, para nombrarse a sí mismas e intentar definir su identi-

70 Cherríe Moraga: The Hungry Woman: A Mexican Medea, en C. Svitch y M. T. Marrero (eds.): Out of the Fringe: Contemporary Latina/Latino Theatre and Performance, pp. 239-363. En España, dos estudios recientes son el de Esther Álvarez López: "The Daughters of the Malinche: Examining the Effects of the Myth in Cherríe Moraga's Work", pp. 51-59, y Martha Fernández Morales, "Revising La Llorona on Stage: Cherríe Moraga's The Hungry Woman: A Mexican Medea", pp. 85-95, ambos en María Herrera Sobek et alt. (eds.): Perspectivas transatlánticas en la Literatura Chicana: Ensayos y creatividad. pp. 49-51.

71 Monica Palacios: "La Llorona Loca: The Other Side", en Trujillo, Chicana Lesbians,

72 Alicia Gaspar de Alba: "The Politics of Location of the Tenth Muse of America: An Interview with Sor Juana Inés de la Cruz", en Trujillo (ed.), Living Chicana Theory, pp. 136-165, p. 144. Otras obras de Gaspar de Alba sobre Sor Juana son "Juana Inés", en Tiffany Ana López (ed.): Growing Up Chicana-o: An Anthology, pp. 69-85; y Sor Juana's Second Dream. 
dad y reivindicar su experiencia, y necesitan hacerlo frente a una serie de acusaciones generales y específicas de traición. Acusadas, como lesbianas y mujeres rebeldes, de traicionar a los hombres al negarse a adoptar el papel reproductor y heterosexual que en teoría corresponde a las mujeres, son acusadas por añadidura de traicionar su identidad como chicanas, ya que el feminismo y el lesbianismo se conciben como implantes "blancos". Como la mayoría de las personas homosexuales, reflejan en su escritura los profundos conflictos con la familia de origen, de la que también reciben acusaciones de traición. Frente a todo esto, los relatos, poemas, novelas y teoría producidos por estas autoras reivindican su derecho a su propia sexualidad, a su libertad como mujeres y al empleo de ideologías liberadoras sea cual sea su procedencia, y a la vez buscan conservar ciertos vínculos con su cultura y su familia de origen, aun sabedoras de que, en palabras de Anzaldúa, son éstas las que las han traicionado a ellas. Con su lúcida crítica de las tradiciones opresivas y su exploración de nuevas formas de existencia y de escritura, la obra de estas autoras contribuye a transformar el pensamiento y la vida dentro del nuevo mundo globalizado.

\section{Bibliografía}

Norma Alarcón: "Making Familia from Scratch: Split Subjectivities in the Work of Helena María Viramontes and Cherríe Moraga", en María Herrera-Sobek and Helena María Viramontes (eds.), Chicana Creativity and Criticism: Charting New Frontiers in American Literature, Houston, Arte Público Press, 1996, pp. 147-159.

- "The Theoretical Subjects of This Bridge Called my Back and AngloAmerican Feminism”, en Héctor Calderón y José David Saldívar (eds.), Criticism in the Borderlands: Studies in Chicano Culture, Durham, Duke University Press, 1991, pp. 28-39.

Flora Alexander: Contemporary Women Novelists, London, Edward Arnold, 1989. Esther Álvarez López: "The Daughters of the Malinche: Examining the Effects of the Myth in Cherríe Moraga's Work", en Herrera Sobek et alt., Perspectivas transatlánticas, pp. 51-59.

Celia Amorós: "Feminismo, multiculturalismo e Ilustración," conferencia pronunciada en la Universidad de Sevilla, 2002. 
Gloria Anzaldúa: “An Interview with Debbie Blake and Carmen Abrego (1994)", en Ana Louise Keating (ed.), Interviews / Entrevistas, New York, Routledge, 2000, pp. 221-233.

- (ed.): Borderlands/La frontera: The New Mestiza, San Francisco, Aunt Lute, 1999.

— "How to Tame a Wild Tongue" en Ibidem, pp. 75-86.

— "La Prieta", en Anzaldúa y Moraga (eds.), This Bridge Called My Back, pp. 220-233.

— "Now let us shift ... the path of conocimiento ... inner work, public acts", en Anzaldúa y Keating (eds.), This bridge we call home, pp. 540-578.

- "The Paisano is a Bird of Good Omen", en Gómez et alt., Cuentos: Stories by Latinas, pp. 153-175.

Gloria Anzaldúa y Ana Louise Keating (eds.): This bridge we call home: radical visions for transformation, New York, Routledge, 2002.

Gloria Anzaldúa y Cherríe Moraga (eds.): This Bridge Called My Back: Writings by Radical Women of Color, New York, Kitchen Table, Women of Color Press, 2002 (1981).

Cathy Arellano: "Lesbiana", en Trujillo (ed.), Chicana Lesbians, pp. 12-13.

Frederick W. Bozett (ed.): Homosexuality and the Family, New York, Howarth, 1989.

F. L. Cardona: "Estudio preliminar", Sor Juana Inés de la Cruz: Poesía Lírica y El divino Narciso, Barcelona, Edicomunicación, 1994.

Ana Castillo: "La Macha: Towards a Beautiful New Self", en Trujillo (ed.), Chicana Lesbians, pp. 24-47.

Sara J. Cervenak, et alt.: "Imagining Differently: The Politics of Listening in a Feminist Classroom”, en Anzaldúa y Keating (ed.), This bridge, pp. 341-356.

Yolanda Chávez Leyva: "Listening to the Silences in Latina/Chicana Lesbian History", en Trujillo (ed.), Living Chicana Theory, pp. 429-434.

Helène Cixous: Sorties: Out and Out: attacks/ways out/forays, selección en Tallack (ed.), Critical Theory, pp. 200-211.

Teresa de Lauretis: "Sexual Indifference and Lesbian Representation", en Henry Abelove et alt., (eds.), The Lesbian and Gay Studies Reader, New York, Routledge, 1993.

Editorial, 23 de abril de 2001. http://www.almalopez.net/ORnews/010423az.html. (11-11-2009).

Lillian Faderman: Chloe plus Olivia: An Anthology of Lesbian Literature from the Seventeenth Century to the Present, Harmondsworth, Penguin, 1995.

- Surpassing the Love of Men, London, The Women's Press, 1991.

Martha Fernández Morales: "Revising La Llorona on Stage: Cherríe Moraga's The Hungry Woman: A Mexican Medea", en Herrera Sobek et alt., Perspectivas transatlánticas, pp. 85-95. 
Rocky Gámez: "From The Gloria Stories”, en Gómez et alt., Cuentos: Stories by Latinas, pp.138-146.

Alicia Gaspar de Alba, Alicia: “Juana Inés”, en Tiffany Ana López (ed.), Growing Up Chicana-o: An Anthology, New York, Williams, Morrow and Co., 1993, pp. 69-85.

- Sor Juana's Second Dream, Albuquerque, New Mexico University Press, 1999. Versión española: El segundo sueño, trad. Bettina Blanch Tyroller, Barcelona, Grijalbo Mondadori, 2001.

- "The Politics of Location of the Tenth Muse of America: An Interview with Sor Juana Inés de la Cruz" en Trujillo (ed.), Living Chicana Theory, pp. 136165.

— “They're just Silly Rabbits", en Sergio D. Elizondo y Armando Armengol (eds.), Palabra nueva: Cuentos chicanos II, El Paso, Dos Pasos, 1987, pp. 107-114.

Alma Gómez et alt. (eds.): Cuentos: Stories by Latinas, New York, Kitchen Table, Women of Color Press, 1983.

Susan M. Guerra: "In the End (Al fin) we are all Chicanas (Somos todos chicanas): pivotal positions for change", en Anzaldúa y Keating (eds.), This bridge we call home, pp. 181-190.

E.D. Hernández: "Discussion, Discourse and Direction: The Dilemmas of a Chicana Lesbian", en Trujillo (ed.), Chicana Lesbians, pp. 138-140.

Manuel de Jesús Hernández-Gutiérrez y David William Foster (eds.): "Introduction", Literatura Chicana 1965-1995: An Anthology in English, Spanish and Caló, New York, Garland, 1997.

María Herrera Sobek et alt. (eds.): Perspectivas transatlánticas en la literatura chicana: Ensayos y creatividad, Málaga, Universidad de Málaga, 2004.

Sheila Jeffreys: La herejía lesbiana, Madrid, Cátedra, 1996.

Naomi Littlebear Morena: "Coming out Queer and Brown", en Sara-Lucia Hoagland y Julia Penelope (eds.), For Lesbians Only: A Separatist Anthology, London, Radical Feminist Lesbian Publishers, 1991, pp. 346347.

— “Dreams of Violence", en Anzaldúa y Moraga (eds.), This Bridge Called my Back, pp. 16-17.

Alma López: "boi hair", http://www.almalopez.net/video/boi.html, (11-11-2009).

Natashia López: "Trying to be Dyke and Chicana", en Trujillo (ed.), Chicana Lesbians, p. 84.

Renée M. Martínez: "Del puente al arco iris: transformando de guerrera a mujer de la paz. From Bridge to Rainbow: Transforming from Warrior to Woman of Peace", en Anzaldúa y Keating (eds.), This bridge we call home, pp. 42-50.

María Moliner: Diccionario de uso del español. Edición abreviada, Madrid, Gredos, 2008. 
Gina Montoya: "Baby Dykes”, en Trujillo (ed.), Chicana Lesbians, pp. 19-20.

Cherríe Moraga: "A Long Line of Vendidas", en Living in the War Years, pp. 90144.

- "Free at Last", en Trujillo (ed.), Living Chicana Theory, pp.166-188.

- Giving up the Ghost, en Heroes and Saints and Other Plays, Albuquerque, West End Press, 1994, pp.1-36.

— "La Güera", en Anzaldúa y Moraga (eds.), This Bridge Called My Back, pp. 24-33.

- "La Ofrenda", en Trujillo (ed.), Chicana Lesbians, pp. 6-9.

- Living in the War Years: Lo que nunca pasó por sus labios, Cambridge, MA, South End Press, 1983.

— "Pesadilla", en Living in the War Years, pp. 36-43.

- The Hungry Woman: A Mexican Medea, en C. Svitch y M. T. Marrero (eds.), Out of the Fringe: Contemporary Latina/Latino Theatre and Performance, New York, Theatre Communications Group, 2000, pp. 239-363.

Marta Navarro: "Interview with Ana Castillo", en Trujillo (ed.), Chicana Lesbians, pp. 113-132.

Julián Olivares: "Entering The House on Mango Street", en John Maitino and David R. Peck (eds.): Teaching American Ethnic Literatures: Nineteen Essays, Albuquerque, New Mexico Press, 1996, pp. 289-228.

Monica Palacios: "La Llorona Loca: The Other Side", en Trujillo (ed.), Chicana Lesbians, pp. 49-51.

- "Personality Fabulosa", en Lillian Castillo-Speed (ed.), Latina: Women's Voices from the Borderlands, New York, Simon and Schuster, 1995, pp. 200207.

— “Tomboy", en Trujillo, Living Chicana Theory, pp. 305-309.

Emma Pérez: "Gulf Dreams", en Trujillo (ed.), Chicana Lesbians, pp. 96-108.

- "Sexuality and Discourse: Notes from a Chicana Survivor", en Ibidem, pp. 159-184.

Immaculada Pertusa-Seva y Lourdes Torres: Tortilleras: Hispanic and U.S. Latina Lesbian Expression, Philadelphia, Temple University Press, 2003.

Estela Portillo Trambley: The Day of the Swallows, en Roberto J. Garza (ed.), Contemporary Chicano Theatre, Notre Dame, University of Notre Dame Press, 1976.

Fred Reed: Nekkid in Austin: Drop your Inner Child Down a Well, Lincoln, Iuniverse, 2002.

Adrienne Rich: "Compulsory Heterosexuality and Lesbian Existence", Signs, vol 5, n. ${ }^{\circ}$ 4, 1980, pp. 631-660.

Bryan E. Robinson et alt:: "Response of Parents to Learning that Their Child Is Homosexual and Concern over AIDS: A National Study", en Bozett, Homosexuality and the Family, pp. 59-80. 
Catriona Rueda Esquibel: With her Machete in her Hand: Reading Chicana Lesbians, Austin, Texas University Press, 2006.

- Bibliography of 20th century Queer Chicana Fictions, http://online.sfsu.edu/ $\sim$ ktrion/Jotas (11-11-2009).

Sonia Saldívar-Hull: "Feminism on the Border: From Gender Politics to Geopolitics", pp. 202-220, en Héctor Calderón y José David Saldívar (eds.): Criticism in the Borderlands: Studies in Chicano Culture, Durham, Duke University Press, 1991.

Juanita M. Sánchez: "Paso a paso", en Trujillo, Chicana Lesbians, p. 15.

Sara Schulman: Ties that Bind: Familial Homophobia and its Consequences, New York, New Press, 2009.

Erik F. Strommen: “'You're a What?': Family Member Reactions to the Disclosure of Homosexuality", en Bozett, Homosexuality and the Family, pp. 37-58.

Douglas Tallack (ed.): Critical Theory: A Reader, New York, HarvesterWheatsheaf, 1995.

Lydia Tirado White: "Intentarás imponerme”, en Trujillo (ed.), Chicana Lesbians, p. 84.

María Ángeles Toda Iglesia: “¿Cuántas cervezas?: Dos versiones de un cuento de Alicia Gaspar de Alba", en Ramón Espejo et alt. (eds.), Critical Essays on Chicano Studies, Bern, Peter Lang, 2004, pp. 245-254.

Carla Trujillo: "Chicana Lesbians: Fear and Loathing in the Chicano Community", en Alma M. García (ed.), Chicana Feminist Thought: The Basic Historical Writings, New York, Routledge, 1997, pp. 281-287.

- (ed.): Chicana Lesbians: The Girls Our Mothers Warned Us About, Berkeley, Third Woman Press, 1991.

- (ed.): Living Chicana Theory, Berkeley, Third Woman Press, 1998.

Alan Unterman: "Judaism and Homosexuality: Some Orthodox Perspectives", en Jonathan Mangonet (ed.), Jewish Explorations of Sexuality, Oxford, Berghahn Books, 1995, pp. 67-74.

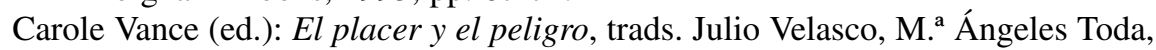
Madrid, Editorial Revolución, 1989.

Monique Wittig: “The Straight Mind”, en Tallack, Critical Theory, pp. 246-252.

Max Wolf Valerio: "It's in My Blood, My Face - My Mother's Voice, The Way I Sweat", en Anzaldúa y Moraga (eds.), This Bridge Called My Back, pp. 4146.

— “"Now that You are a White Man': Changing Sex in a Postmodern WorldBeing, Becoming, and Borders", en Anzaldúa y Keating (eds.), This bridge we call home, pp. 239-254. 